\title{
Heyting Valued Set Theory and Sato Hyperfunctions
}

\author{
By
}

\author{
Hirokazu NISHIMURA*
}

\begin{abstract}
Following the lines of Takeuti and Titani [16] as well as Rousseau [9], this paper shows that Sato hyperfunctions with holomorphic parameters can be viewed as those without parameters in an appropriately chosen intuitionistic universe, where we establish, as an application of this idea, de Rham and Dolbeault theorems with hyperfunctional coefficients.
\end{abstract}

\section{§1. Preamble}

Heyting valued set theory, which is a natural generalization of Boolean valued set theory, enjoys its close relationship to modern mathematics and analysis in particular. Indeed, as was demonstrated by Takeuti and Titani [16] as well as Rousseau [9], an intuitionistic viewpoint enables us to treat entities with parameters as if they were without parameters in a constructive universe based on the topological space of parameters. By way of example, vector bundles and families of complex structures over a complex manifold $M$ are apartness vector spaces and complex manifolds respectively in a constructive universe based on the topological structure of $M$.

By the way, in hyperfunction theory invented by Sato $[11,12]$, hyperfunctions with holomorphic parameters play a significant role and we will show in Sections 3 and 4, after reviewing some rudiments of Heyting valued set theory, that they can be treated on the lines of Takeuti and Titani [16] as well as Rousseau [9]. This viewpoint

Received March 3, 1986.

* Research Institute for Mathematical Sciences, Kyoto University, Kyoto 606, Japan.

Present address : Institute of Mathematics, University of Tsukuba, Sakura-mura, Niiharigun, Ibaraki-ken 305, Japan. 
enables us to derive somewhat restricted de Rham and Dolbeault theorems with hyperfunctional coefficients in a constructive universe from the well-known de Rham and Dolbeault resolutions with holomorphic parameters, as will be demonstrated in Section 5. The last section is devoted to presenting some topics for future study. A little more effort would enable us to formulate our idea in a more manifold-oriented formalism, which is left to the interested and competent reader.

\section{§2. Heyting Valued Set Theory}

In this section we review some rudiments of Heyting valued set theory of Takeuti and Titani [14].

\subsection{Intuitionistic Set Theory}

By $\mathrm{ZF}_{\mathrm{I}}$ we mean a first-order intuitionistic theory with a unary relation symbol $\mathrm{E}$ and two binary relation symbols $\in$ and $=$ satisfying the following nonlogical axioms:

(Al) Equality axioms: $u=u$,

$$
\begin{aligned}
& u=v \rightarrow v=u, \\
& u=v \wedge \varphi(u) \rightarrow \varphi(v), \text { and }(\mathrm{E} u \bigvee \mathrm{E} v \rightarrow u=v) \rightarrow u=v .
\end{aligned}
$$

(A2) Extensionality: $\dot{\forall} z(z \in u \leftrightarrow z \in v) \wedge(\mathrm{E} u \leftrightarrow \mathrm{E} v) \rightarrow u=v$.

(A3) Pairing: $\dot{\exists} z \dot{\forall} x(x \in z \leftrightarrow x=u \bigvee x=v)$.

(A4) Union: $\dot{\exists} v \dot{\forall} x(x \in v \leftrightarrow \dot{\exists} y \in u(x \in y))$.

(A5) Power sets: $\dot{\exists} v \dot{\forall} x(x \in v \leftrightarrow \dot{\forall} y \in u(y \in x))$.

(A6) $\in$-induction: $\dot{\forall} x(\dot{\forall} y \in x \varphi(y) \rightarrow \varphi(x)) \rightarrow \dot{\forall} x \varphi(x)$.

(A7) Infinity: $\dot{\exists} v(\dot{\exists} x \in v \wedge \dot{\forall} x \in v \dot{\exists} y \in v(x \in y))$.

(A8) Separation: $\dot{\exists} v \dot{\forall} x(x \in v \leftrightarrow x \in u \wedge \varphi(x))$.

(A9) Collection: $\dot{\exists} v(\dot{\forall} x \in u \dot{\exists} y \varphi(x, y) \rightarrow \dot{\forall} x \in u \dot{\exists} y \in v \varphi(x, y))$.

In the above list $\dot{\forall} x \ldots$ and $\dot{\exists} x \ldots$ are abbreviations of $\forall x$ $(\mathrm{E} x \rightarrow \cdots)$ and $\exists x(\mathrm{E} x \wedge \cdots)$. Since $\forall x$ and $\exists x$ will usually appear in these forms, we will often write $\forall x$ and $\exists x$ simply for $\dot{\forall} x$ and $\dot{\exists} x$. 


\section{2. Heyting Valued Models}

Let $V$ be a standard universe of ZFG and let $\Omega$ be a complete Heyting algebra. For each ordinal $\alpha$ we define $V_{\alpha}^{(\Omega)}$ inductively to be the set of all ordered pairs $\langle u, \mathrm{E} u\rangle$ such that:

(1) $\mathrm{E} u \in \Omega$;

(2) $\quad u$ is an $\Omega$-valued function defined on a subset $\mathscr{D}(u)$ of $V_{\beta}^{(\Omega)}$ for some ordinal $\beta<\alpha$;

$$
\forall x \in \mathscr{D}(u)(u(x) \leqq \mathrm{E} u \wedge \mathrm{E} x) \text {. }
$$

Now $V^{(\Omega)}$ is defined to be the class $\cup_{\alpha \in \mathrm{On}} V_{\alpha}^{(\Omega)}$, which is to be called an $(\Omega$-valued) sheaf model, can be considered to be a Heyting valued model of $\mathrm{ZF}_{\mathrm{I}}$ by defining [Eu] with

$$
\llbracket \mathrm{E} u \rrbracket=\mathrm{E} u,
$$

and by defining $[u \in v \rrbracket$ and $[u=v \rrbracket$ with the following simultaneous induction

$$
\begin{aligned}
& \llbracket u \in v \rrbracket=\bigvee_{y \in \mathscr{D}(v)}(v(y) \wedge \llbracket u=y \rrbracket), \\
& \llbracket u=v \rrbracket=\bigwedge_{x \in \mathscr{D}(u)}\left(u(x) \rightarrow \llbracket x \in v \rrbracket \wedge \wedge_{y \in \mathscr{D}(v)}(v(y) \rightarrow \llbracket y \in u \rrbracket) \wedge(\mathrm{E} u\right. \\
& \leftrightarrow \mathrm{E} v),
\end{aligned}
$$

and then by assigning a Heyting value $\llbracket \varphi \rrbracket$ to each nonatomic sentence $\varphi$ inductively as follows:

(4) $\quad \llbracket \varphi_{1} \wedge \varphi_{2} \rrbracket=\llbracket \varphi_{1} \rrbracket \wedge \llbracket \varphi_{2} \rrbracket$,

(5) $\quad \llbracket \varphi_{1} \vee \varphi_{2} \rrbracket=\llbracket \varphi_{1} \rrbracket \vee \llbracket \varphi_{2} \rrbracket$,

(6) $\quad\left[\varphi_{1} \rightarrow \varphi_{2} \rrbracket=\llbracket \varphi_{1} \rrbracket \rightarrow \llbracket \varphi_{2} \rrbracket\right.$,

(7) $\quad[\neg \varphi \rrbracket=\neg \llbracket \varphi \rrbracket$,

(8) $\quad \llbracket \exists x \varphi(x) \rrbracket=V_{x \in V^{(s)}} \llbracket \varphi(x) \rrbracket$,

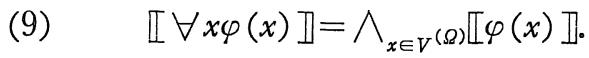

Now we have

Theorem 2.2.1. $V^{(\Omega)}$ is a model of $\mathrm{ZF}_{\mathrm{I}}$.

The class $V$ can be embedded into $V^{(\Omega)}$ by transfinite induction 
as follows:

$$
\check{y}=\{\langle\check{x}, 1\rangle \mid x \in y\} \text { and } \mathrm{E} \check{y}=1 \quad \text { for } y \in V .
$$

For $u \in V^{(\Omega)}$ and $p \in \Omega$, we define $u\left\lceil p\right.$ to be the element of $V^{(\Omega)}$ such that

$$
\begin{aligned}
& \mathscr{D}(u\lceil p)=\{x \Gamma p \mid x \in \mathscr{D}(u)\}, \\
& (u\lceil p)(x\lceil p)=\bigvee\{u(t) \wedge p \mid t \in \mathscr{D}(u), t\lceil p=x \Gamma p\} \text { for } x \in \mathscr{D}(u), \\
& \text { and } \mathrm{E}(u\lceil p)=\mathrm{E} u \wedge p .
\end{aligned}
$$

In the sequel we implicitly identify $x, y \in V^{(\Omega)}$ time and again provided $[x=y]=1$.

\section{3. Sheaves over Complete Heyting Algebras}

A presheaf over a complete Heyting algebra $\Omega$ is a triple $\langle S, \mathrm{E}, \Gamma\rangle$ of a set $S$ and two functions $\mathrm{E}: S \rightarrow \Omega$ and $\Gamma: S \times \Omega \rightarrow S$ with the following properties:

$$
\begin{aligned}
& a\ulcorner 0=b \Gamma 0, \\
& a \Gamma \mathrm{E} a=a, \\
& \mathrm{E}(a \Gamma p)=\mathrm{E} a \wedge p, \\
& (a \Gamma p) \Gamma q=a \Gamma(p \wedge q) .
\end{aligned}
$$

For convenience we often say simply that $S$ is a presheaf over $\Omega$ without mentioning $\mathrm{E}$ and $\Gamma$ explicitly. Members $a, b$ of a presheaf $S$ over $\Omega$ are said to be compatible whenever $a \digamma \mathrm{E} b=b \Gamma \mathrm{E} a$. A subset $F$ of $S$ whose members are pairwise compatible is called compatible. A presheaf $S$ over $\Omega$ is called a sheaf over $\Omega$ if for any compatible subset $F$ of $S$ there exists a unique $g \in S$ such that:

(1) $f \in F$ implies $g\ulcorner\mathrm{E} f=f$,

(2) $\quad \mathrm{E} g=\bigvee\{\mathrm{E} f \mid f \in F\}$.

The subset $\{a \in S \mid \mathrm{E} a=p\}$ is denoted by $\Gamma(p, S)$.

Theorem 2.3.1. For any $u \in V^{(\Omega)}, \tilde{u}=\left\{x \in V^{(\Omega)} \mid[x \in u \rrbracket=\mathrm{E} x\}\right.$ is a sheaf to be called the sheaf represented by $u$. Conversely, for any sheaf 
$S$ over $\Omega$, there is an element $u \in V^{(\Omega)}$ such that the sheaf $\tilde{u}$ represented by $u$ is isomorphic to $S$.

Similarly we have

Theorem 2. 3. 2. Let $u_{1}, u_{2} \in V^{(\Omega)}$. Then any function $f: u_{1} \rightarrow u_{2}$ in $V^{(\Omega)}$ renders a unique function $\tilde{f}: \tilde{u}_{1} \rightarrow \tilde{u}_{2}$ such that for each $a \in \tilde{u}_{1}$,

$$
\mathrm{E}_{\tilde{u}_{1}} a=\mathrm{E}_{\tilde{u}_{2}} \tilde{f}(a)=\mathbb{L}\langle a, \tilde{f}(a)\rangle \in f \rrbracket .
$$

This gives a bijective correspondence between functions $f: u_{1} \rightarrow u_{2}$ in $V^{(\Omega)}$ and functions $g: \tilde{u}_{1} \rightarrow \tilde{u}_{2}$ such that

$$
\begin{aligned}
& \mathrm{E}_{\tilde{u}_{1}} a=\mathrm{E}_{\tilde{u}_{2}} g(a) \text { and } g(a) \Gamma p=g(a \Gamma p) \\
& \text { for any } p \in \Omega \text { and } a \in \tilde{u}_{1} .
\end{aligned}
$$

\subsection{Real-Like and Complex-Like Fields}

Let $V$ be a standard universe of ZFG and let $\Omega$ be a complete Heyting algebra. Then the set $\mathbb{R}^{(\Omega)}$ of all real numbers in $V^{(\Omega)}$ is defined to be the set of all ordered pairs $\langle L, U\rangle$ of subsets of $Q$ such that

(1) $\exists r, s \in \check{\mathbb{Q}}(s \in L \wedge r \in U)$,

(2) $\forall r \in \check{Q}\urcorner(r \in U \wedge r \in L)$,

(3) $\forall r \in \check{Q}(r \in U \leftrightarrow \exists s \in \check{Q}(s<r \wedge s \in U))$,

(4) $\forall r \in \check{Q}(r \in L \leftrightarrow \exists s \in \check{Q}(r<s \wedge s \in L))$,

(5) $\forall r, s \in \grave{Q}(s<r \rightarrow s \in L \bigvee r \in U)$.

A subset $\mathfrak{R}$ of $\mathbb{R}^{(\Omega)}$ is called a real-like field if

(1) $\quad \Re$ contains $\check{\mathscr{R}}$,

(2) $\quad \Re$ is an apartness subfield of $\mathbb{R}^{(\Omega)}$, and

(3) $\Re$ is complete, i. e., every Cauchy sequence of $\Re$ converges to an element of $\Re$ 。

Let $\mathbb{C}^{(\Omega)}=\mathbb{R}^{(\Omega)}+i \mathbb{R}^{(\Omega)}$, as is expected. Then the notion of a complex-like field $\mathfrak{E}$ is defined similarly. 


\section{§3. Relative Cohomology with Sheaf Coefficients in $V^{(\Omega)}$}

Throughout this section, let $V^{(\Omega)}$ be a sheaf model of $\mathrm{ZF}_{\mathrm{I}}$ with a standard universe $V$ of ZFG and a complete Heyting algebra $\Omega$ in $V$. Then we have

Theorem 3.1. Any complete Heyting algebra $\theta$ in $V^{(\Omega)}$ is also externally a complete Heyting algebra. I. e., $\tilde{\theta}\left(=\left\{a \in V^{(\Omega)} \mid \llbracket a \in \theta \rrbracket=\mathrm{E} a\right\}\right)$ is a complete Heyting algebra in $V$.

Proof. See Takeuti and Titani [13; Chapter I, Theorem 4].

Theorem 3.2. Any sheaf $\mathscr{F}=\langle S, \mathrm{E}, \Gamma\rangle$ over a complete Heyting algebra $\theta$ in $V^{(\Omega)}$ is externally a sheaf $\tilde{\mathscr{F}}=\langle\tilde{S}, \tilde{\mathrm{E}}, \tilde{\Gamma}\rangle$ over $\tilde{\theta}$.

Proof. Follows easily from Theorem 2.3.2.

Given a sheaf $\mathscr{F}$ of moduli over a complete Heyting algebra $\theta$ and $p, q \in \theta$ with $q \leqq p$, the notion of relative cohomology moduli

$$
H^{i}(p \bmod q, \mathscr{F})(i=0,1,2, \ldots)
$$

with coefficients in $\mathscr{F}$ can be defined by imitating the usual construction of relative Čech cohomology with sheaf coefficients, just as Takeuti and Titani $[16 ; 2.3]$ did for absolute cohomology. And we have

Theorem 3.3. Let $\mathscr{F}$ be a sheaf of moduli over a complete Heyting algebra $\theta$ and $p, q \in \theta$ with $q \leqq p$ in $V^{(\Omega)}$. Then the cohomology moduli $H^{i}(p \bmod q, \mathscr{F})(i=0,1,2, \ldots)$ are externally the sheaves determined by the presheaves

$$
r \in \Omega \mapsto H^{i}(p\lceil r \bmod q\ulcorner r, \tilde{\mathscr{F}}) .
$$

Proof. Follows from the definition of $H^{i}(p \bmod q, \mathscr{F})$.

\section{§4. Sato Hyperfunctions in $V^{(\Omega)}$}

The three theorems of the previous section have more concrete 
descriptions when we deal with specific complete Heyting algebras often encountered in analysis.

Theorem 4. 1 . If $\Omega$ is the complete Heyting algebra of open sets of $\mathbb{C}^{n}$, then, for any complex-like field $\sqrt{5}$ in $V^{(\Omega)}$, the totality of open sets of $\mathfrak{C}^{m}$ is essentially the same and it is externally the totality of open sets of $\mathbb{C}^{m+n}$. Similarly, for any real-like field $\Re$ in $V^{(\Omega)}$, the totality of open sets of $\Re^{m}$ is essentially the same and it is externally the totality of open sets of $\mathbb{R}^{m} \times \mathbb{C}^{n}$.

Proof. See Rousseau [9] and Takeuti [13; Chapter 3, §3].

Due to this theorem, when we consider a sheaf on $\mathfrak{R}^{m}$ (or on $\mathfrak{5}^{m}$ ), it is not essential to specify $\mathfrak{R}$ (or $(5)$ ) among various real-like (or complex-like) fields.

In the sequel, for any open set $S$ of $\mathbb{R}^{m}, S^{(\Omega)}$ denotes the open set of a real-like field in $V^{(\Omega)}$ corresponding to $S \times \mathbb{C}^{n}$ under Theorem 4.1.

Theorem 4.2. If $\Omega$ is the complete Heyting algebra of open sets of $C^{n}$, then the sheaf $\mathcal{O}_{m}$ of holomorphic functions of $m$ variables in $V^{(\Omega)}$, where function theory is developed using the complex-like field of all holomorphic function of $n$ variables, is externally the sheaf $\mathcal{O}_{m+n}$ of holomorphic functions of $m+n$ variables.

Proof. See Rousseau [9] and Takeuti [13; Chapter 3, §3].

In $\mathrm{ZFG}$ the sheaf $\mathscr{B}_{m}$ of hyperfunctions of $m$ variables on $\mathbb{R}^{m}$ derives from the sheaf

$$
S \mapsto H^{m}\left(S \bmod S-\mathbb{R}^{m}, \mathcal{O}_{m}\right)
$$

on $\mathbb{C}^{m}$, since relative cohomology enjoys the excision propert $f_{0}$ This notion survives in $V^{(\Omega)}$. And we have

Theorem 4.3. If $\Omega$ is the complete Heyting algebra of open sets of $\mathbb{C}^{n}$, then the sheaf $\mathscr{B}_{m}$ of hyperfunctions of $m$ variables in $V^{(\Omega)}$ is externally the sheaf $\mathscr{B}_{m} \mathcal{O}_{n}$ of hyperfunctions of $m$ variables with $n$ holomorphic parameters, which derives from the sheaf

$$
S \mapsto H^{m}\left(S \bmod S-\mathbb{R}^{m} \times \mathbb{C}^{n}, \mathcal{O}_{m+n}\right)
$$


Proof. Follows from Theorems 3.3 and 4.2.

\section{§5. De Rham and Dolbeault Theorems with Hyperfunctional Coefficients in $V^{(\Omega)}$}

In ZFG de Rham theorem with hyperfunctional coefficients and no parameters follows readily from the exact sequence (so-called de Rham resolution)

$$
0 \longrightarrow \mathscr{C}_{m} \longrightarrow \mathscr{B}_{m}^{(0)} \stackrel{d}{\longrightarrow} \mathscr{B}_{m}^{(1)} \stackrel{d}{\longrightarrow} \cdots \stackrel{d}{\longrightarrow} \mathscr{B}_{m}^{(m)} \longrightarrow 0,
$$

where $\mathscr{C}_{m}$ is the complex-valued constant sheaf on $\mathbb{R}^{m}$ and $\mathscr{B}_{m}^{(i)}$ is the sheaf of $i$-forms with hyperfunctional coefficients since the sheaf $\mathscr{B}_{m}$ is flabby. Indeed we have

$$
H^{i}\left(S, \mathscr{C}_{m}\right)=\frac{\Gamma\left(S, d \mathscr{B}_{m}^{(i-1)}\right)}{d \Gamma\left(S, \mathscr{B}_{m}^{(i-1)}\right)}
$$

for any open subset $S$ of $\mathbb{R}^{m}$ and $i \geqq 1$.

The de Rham resolution (I) is easily generalized to the exact sequence with $n$ holomorphic parameters

(II) $\quad 0 \longrightarrow \mathscr{C}_{m} \mathcal{O}_{n} \longrightarrow \mathscr{B}_{m} \mathcal{O}_{n}^{(0)} \stackrel{d}{\longrightarrow} \mathscr{B}_{m} \mathcal{O}_{n}^{(1)} \stackrel{d}{\longrightarrow} \cdots \stackrel{d}{\longrightarrow} \mathscr{B}_{m} \mathcal{O}_{n}^{(m)} \longrightarrow 0$,

where $\mathscr{C}_{m} \mathcal{O}_{n}$ is the complex-valued constant sheaf (of the first $m$ variables) with $n$ holomorphic parameters (on $\mathbb{R}^{m} \times \mathbb{C}^{n}$ ) and $\mathscr{B}_{m} \mathscr{O}_{n}^{(i)}$ is the sheaf of $i$-forms with hyperfunctional coefficients (of the first $m$ variables) and $n$ holomorphic parameters. Although $\mathscr{B}_{m} \mathcal{O}_{n}$ is flabby merely with respect to the first $m$ variables (strictly speaking, the sheaf

$$
S \mapsto \mathscr{B}_{m} \mathcal{O}_{n}(S \times T)
$$

on $\boldsymbol{R}^{m}$ is flabby provided $T$ is a Stein open subset of $\boldsymbol{C}^{n}$ ), a bit subtler argument gives

Theorem 5.1. If $\Omega$ is the complete Heyting algebra of open sets of $\boldsymbol{C}^{n}$, then we have the following de Rham theorem with hyperfunctional coefficients in $V^{(\Omega)}$.

$$
H^{i}\left(S^{(\Omega)}, \boldsymbol{C}_{m}\right)=\frac{\Gamma\left(S^{(\Omega)}, d \mathscr{B}_{m}^{(i-1)}\right)}{d \Gamma\left(S^{(\Omega)}, \mathscr{B}_{m}^{(i-1)}\right)}
$$


for any open subset $S$ of $\mathbb{R}^{m}$ and $i \geqq 1$.

Proof. Since the sheaves $\mathscr{C}_{m}$ and $\mathscr{B}_{m}^{(i)}$ in $V^{(\Omega)}$ are externally the sheaves $\mathscr{C}_{m} \mathcal{O}_{n}$ and $\mathscr{B}_{m} \mathcal{O}_{n}^{(i)}$, it is sufficient to show that

$$
\begin{aligned}
& T \mapsto H^{i}\left(S \times T, \mathscr{C}_{m} \mathcal{O}_{n}\right) \quad \text { and } \\
& T \mapsto \frac{\Gamma\left(S \times T, d \mathscr{B}_{m} \mathcal{O}_{n}^{(i-1)}\right)}{d \Gamma\left(S \times T, \mathscr{B}_{m} \mathcal{O}_{n}^{(i-1)}\right)}
\end{aligned}
$$

determine the same sheaf on $\boldsymbol{C}^{n}$. However, since the sheaf on $\mathbb{R}^{m}$

$$
U \mapsto \mathscr{B}_{m} \mathcal{O}_{n}(U \times T)
$$

is flabby for any Stein open subset $T$ of $\mathbb{C}^{n}$, we have

$$
\begin{aligned}
H^{i}\left(S \times T, \mathscr{C}_{m} \mathcal{O}_{n}\right) & =H^{i}\left(S, \mathscr{C}_{m} \mathscr{O}_{n}({ } \times T)\right) \\
& =\frac{\Gamma\left(S \times T, d \mathscr{B}_{m} \mathcal{O}_{n}^{(i-1)}\right)}{d \Gamma\left(S \times T, \mathscr{B}_{m} \mathcal{O}_{n}^{(i-1)}\right)},
\end{aligned}
$$

where $\mathscr{C}_{m} \mathcal{O}_{n}\left(\_\times T\right)$ is the sheaf on $\mathbb{R}^{m}$ assigning $\mathscr{C}_{m} \mathcal{O}_{n}(U \times T)$ to each open subset $U$ of $\mathbb{R}^{m}$. Since each point $x$ of $\mathbb{C}^{n}$ has a basic neighborhood system consisting of Stein open sets, we have

$$
\begin{aligned}
& \stackrel{\lim }{\longrightarrow}\left\{H^{i}\left(S \times T, \mathscr{C}_{m} \mathcal{O}_{n}\right) \mid T \in \mathfrak{O}_{x}\right\} \\
& =\underset{\lim }{\longrightarrow}\left\{H^{i}\left(S \times T, \mathscr{C}_{m} \mathcal{O}_{n}\right) \mid T \in S_{S} ._{x}\right\} \\
& =\underline{\lim }\left\{\frac{\Gamma\left(S \times T, d \mathscr{B}_{m} \mathcal{O}_{n}^{(i-1)}\right)}{d \Gamma\left(S \times T, \mathscr{B}_{m} \mathcal{O}_{n}^{(i-1)}\right)} \mid T \in \mathcal{S S}_{x}\right\} \\
& =\lim _{\longrightarrow}\left\{\frac{\Gamma\left(S \times T, d \mathscr{B}_{m} \mathcal{O}_{n}^{(i-1)}\right)}{d \Gamma\left(S \times T, \mathscr{B}_{m} \mathcal{O}_{n}^{(i-1)}\right)} \mid T \in \mathfrak{O}_{x}\right\},
\end{aligned}
$$

where $\mathfrak{S}_{x}$ is the totality of open subsets of $\mathbb{C}^{n}$ containing $x$ and $\mathfrak{S}_{x}$ is the totality of Stein open subsets of $\mathbb{C}^{n}$ containing $x$. Thus the desired conclusion follows.

Similarly, just as in ZFG the exact sequence (so-called Dolbeault resolution)

$$
0 \longrightarrow \mathcal{O}_{m} \longrightarrow \mathscr{B}_{2 m}^{(0,0)} \stackrel{\bar{\partial}}{\longrightarrow} \mathscr{B}_{2 m}^{(0,1)} \stackrel{\bar{\partial}}{\longrightarrow} \ldots \stackrel{\bar{\partial}}{\longrightarrow} \mathscr{\mathscr { B }}_{2 m}^{(0, m)} \longrightarrow 0
$$

with $\mathscr{B}_{2 m}^{(0, i)}$ being the sheaf of $(0, i)$-forms with hyperfunctional coefficients and $\mathbb{C}^{m}$ being regarded as $\boldsymbol{R}^{2 m}$ renders the Dolbeault theorem

$$
H^{i}\left(S, \mathcal{O}_{m}\right)=\frac{\Gamma\left(S, \bar{\partial} \mathscr{B}_{2 m}^{(0, i-1)}\right)}{\bar{\partial} \Gamma\left(S, \mathscr{B}_{2 m}^{(0, i-1)}\right)}
$$

for any open subset $S$ of $\mathbb{R}^{m}$ and $i \geqq 1$, the exact sequence with $n$ 
holomorphic parameters

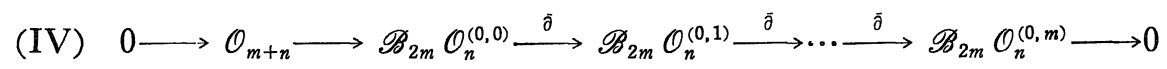

with $\boldsymbol{C}^{m}$ being regarded as $\boldsymbol{R}^{2 m}$ and $\mathscr{B}_{2 m} \mathcal{O}_{n}^{(0, i)}$ being the sheaf of $(0, i)$-forms with hyperfunctional coefficients (of the first $2 m$ real variables) and $n$ holomorphic parameters gives

Theorem 5.2. If $\Omega$ is the complete Heyting algebra of open sets of $\mathbb{C}^{n}$, then we have the following Dolbeault theorem in $V^{(\Omega)}$.

$$
H^{i}\left(S^{(\Omega)}, \mathcal{O}_{m}\right)=\frac{\Gamma\left(S^{(\Omega)}, \bar{\partial} \mathscr{B}_{2 m}^{(0, i-1)}\right)}{\bar{\partial} \Gamma\left(S^{(\Omega)}, \mathscr{B}_{2 m}^{(0, i-1)}\right)}
$$

for any open subset $S$ of $\boldsymbol{C}^{m}$, and $\mathfrak{C}$ is a complex-like field of the form $\mathfrak{E}=\mathfrak{\Re}+i \Re$ with a real-like field $\mathfrak{R}$ and $i \geqq 1$.

Proof. Proceed as in Theorem 5. 1.

\section{§6. Concluding Remarks}

The notion of a hyperfunction depends essentially on that of a holomorphic function and our notion of a holomorphic function in $V^{(\Omega)}$ with $\Omega$ being the complete Heyting algebra of open sets of $\mathbb{C}^{n}$ was supposed to be developed using the complex-like field of all holomorphic functions of $n$ variables. Since any complex-like field can afford function theory as discussed in Rousseau [9] and Takeuti [13], it would be interesting to investigate how the choice of a complex-like field affects the notion of a hyperfunction in $V^{(\Omega)}$.

Another interesting topic for future study is to develop a constructive version of hyperfunction theory, e. g., after the manner of Bishop and Bridges [1]. Related to this topic, a constructive approach to cohomological properties of holomorphic functions and vanishing theorems in particular is highly recommended to try.

Last but not least, hyperfunction theory invented by Sato has grown up to be what is now called algebraic analysis, one of the most actively studied and apparently most promising areas of modern analysis, for which the reader is referred, e. g., to Kashiwara et al. [7]. The interconnection between Heyting valued set theory and algebraic analysis appears to be an intriguing but stiff subject for future study. 


\section{References}

[1] Bishop, E. and Bridges, D., Constructive analysis, Springer, Berlin, 1985.

[2] Grayson, R. J., Concepts of general topology in constructive mathematics and in sheaves, Annals of Mathematical Logic, 20 (1981), 1-41.

[3] , Concepts of general topology in constructive mathematics and in sheaves, II, Annals of Mathematical Logic, 23 (1982), 55-98.

[4] Gunning, R. C. and Rossi, H., Analytic functions of several complex variables, Prentice-Hall, Englewood Cliffs, 1965.

[5] Johnstone, P. T., Topos theory, Academic Press, London, 1977.

[6] Kaneko, A., An introduction to hyperfunctions (in Japanese), 2 vols., University of Tokyo Press, Tokyo, 1980 and 1982.

[ 7 ] Kashiwara, M., Kawai, T. and Kimura, T., Foundations of algebraic analysis (in Japanese), Kinokuniya, Tokyo, 1980.

[8] Morimoto, M., An introduction to Sato hyperfunctions (in Japanese), Kyoritsu, Tokyo, 1976.

[9] Rousseau, C., Topos theory and complex analysis, Lecture Notes in Mathematics, 753 (1977), Springer, Berlin, 623-659.

[10] Ruitenberg, W. B. G., Intuitionistıc algebra, theory and sheaf models, dissertation, 1982.

[11] Sato, M., On a generalization of the concept of functions, II, Proc. Japan Acad., 34 (1958), 604-608.

[12] - Theory of hyperfunctions, II, J. Fac. Sci. Univ. Tokyo, Sec. I, 8 (1960), 387-437.

[13] Takeuti, G., Intuitionistic set theory (in Japanese), Kinokuniya, Tokyo, 1980.

[14] Takeuti, G. and Titani, S., Heyting valued universes of intuitionistic set theory, Lecture Notes in Mathematics, 891 (1980), Springer, Berlin, 189-306.

[15] Globalization of intuitionistic set theory, preprint.

[16] Global intuitionistic analysis, preprint. 
